\title{
SMASH PRODUCTS AND $G$-GALOIS ACTIONS
}

\author{
JAMES OSTERBURG
}

\author{
Dedicated to Professor Hisao Tominaga on his sixtieth birthday
}

\begin{abstract}
We show that duality for coactions follows because the smash product is a $G$-Galois extension. We study $X$-inner and $X$-outer actions of the smash product and prove that if $A$ is a semiprime $G$-graded ring such that $G$ is $X$-outer on the smash product then the center of $A$ is contained in the homogeneous component of the identity element of $G$.
\end{abstract}

1. Introduction. In this paper we discuss the smash product of a $G$-graded $k$-algebra $A$ and the Hopf algebra $k G^{*}$, the dual of the group algebra $k G$, with $G$ finite. We will denote the smash product by $A \# k G^{*}$. Now $G$ acts on $A \# k G^{*}$ with fixed ring $A$, as was observed in [1]. In this paper, we note that this is a $G$-Galois action, which was studied in the 1960s. This is a "module theoretic" approach to Galois theory. So in $\S 1$, we define the concepts mentioned above and show why a result of Cohen-Montgomery, the Duality for Coactions Theorem [1, Theorem 3.5], follows. Also, M. van den Bergh has shown that if $H$ is a Hopf algebra and $A$ an $H$ module algebra [1, Definition 1.1], then $A \# H / A$ is $H$-Galois [7, Theorem 4].

More recently, Galois theory has become much ring theoretic in nature. This applies in particular to the papers of Kharchenko, and to his successors. We take these results as our starting place, and our Theorem 3 is what allows us to use the theorems in [3].

We next calculate the maximal and the Martindale ring of quotients of $A$, a semiprime $G$-graded ring. Finally, for such rings $A$, we assume the $X$-inner automorphisms of $A \# k G^{*}$ which are in $G$ form a subgroup $H$ of $G$. We then show that the center of $A$ is $H$-homogeneous.

I would like to thank Professors M. Cohen, S. Montgomery and the referee for their useful suggestions and corrections.

1. Galois actions and duality. Let $G$ be a finite group and $A$ be a $k$ algebra with 1 , where $k$ is a commutative ring, such that $A$ is a $G$-graded ring, say $A=\sum_{G} A_{g}$ (direct), $A_{g} A_{h} \leqslant A_{g h}$ for $g, h$ in $G$, where $A_{g}$ is an additive subgroup of $A$ for all $g \in G$. If $A_{g} A_{h}=A_{g h}$, we say $A$ is strongly $G$-graded. Let $k G^{*}$ be the dual algebra of $k G$, the group ring. We briefly describe the smash product of $A$ and $k[G]^{*}$. We

Received by the editors July 29, 1985 and, in revised form, September 25, 1985. Presented to the 92nd Annual Meeting of the AMS, January 7-11, 1986.

1980 Mathematics Subject Classification (1985 Revision). Primary 16A03, 16A74; Secondary 16A72, $16 \mathrm{~A} 24$. 
follow the notation in [1] and the reader should consult it for complete details. Now $\left\{p_{g} \mid g\right.$ in $\left.G\right\}$, where $p_{g}$ is the projection onto the $g$ th coefficient, is a $k$ basis of $k[G]^{*}$. What follows is [1, Proposition 1.4]. The smash product $A \# k[G]^{*}$ is the free left and right $A$ module with basis $\left\{1 \# p_{g} \mid g\right.$ in $\left.G\right\}$, a set of orthogonal idempotents whose sum is 1 , and with multiplication given by $\left(a \# p_{g}\right)\left(b \# p_{h}\right)=a\left(b_{g h^{-1}}\right) \# p_{h}$, where $b_{g}$ is the $g$ th part of $b$.

Also very briefly we describe $G$-Galois actions. Our source is [2]. We let $R$ be any ring with a 1 and $G$ a finite group of ring automorphisms that act on $R$. We form the skew group ring $R * G$ and the fixed ring $R^{G}$ as in [2]. We regard $R$ as a left $R * G$ module and a right $R^{G}$ module. The next result is [2, Theorem 1].

THEOREM 1. The following are equivalent:

A. $R$ is finitely generated projective as a right $R^{G}$ module and $j: R * G$ to $\operatorname{End}_{R^{G}}(R)$ where $j\left(\sum_{G} r g\right)(x)=\Sigma_{G} r x^{g}$ for $r, x \in R$ and $g \in G$ is an isomorphism of rings.

B. There are elements in $R$ (called a Galois base) $a_{1}, \ldots, a_{t}, b_{1}, \ldots, b_{t}$ such that $\sum a_{i} b_{i}^{g}=1$ if $g=1$ and the sum is 0 if $g \neq 1$.

We say $G$ is a G-Galois action, if A or $\mathrm{B}$ is satisfied.

We note the inverse of $j$ is $j^{-1}(f)=\sum_{i, g} f\left(a_{i}\right)\left(b_{i}^{g}\right) g$, for $f$ in $\operatorname{End}_{R^{G}} R$.

If $G$ is a $G$-Galois action and there is an element $d$ in $R$ such that the trace of $d=\operatorname{tr}(d)=\Sigma_{G} d^{g}=1\left(\Leftrightarrow R^{G}\right.$ is an $R^{G}$ direct summand of $\left.R\right)$, then $R * G$ is Morita equivalent to $R^{G}$. So as usual, there is a bijection between the two-sided ideals of $R * G$ and $R^{G}$.

We return to the smash product and following [1], we let $G=\{1, \ldots, k\}$ act on $R=A \# k[G]^{*}$ by $\left(a \# p_{h}\right)^{g}=a \# p_{h g}$. This is a $G$-Galois action for our Galois base: take $a_{1}=1 \# p_{1}, \ldots, a_{k}=1 \# p_{k} ; b_{1}=1 \# p_{1}, \ldots, b_{k}=1 \# p_{k}$ where $G=\{1, \ldots, k\}$. Since these idempotents are orthogonal and sum to 1 they form a Galois base of $R$ over $R^{G}=A$.

The next theorem was proven by Cohen-Montgomery [1, Theorem 3.5]. Let $\operatorname{Mat}_{n}(A)$ be the ring of $n \times n$ matrices over $A$ where $n=|G|$.

TheOREM 2 (DUALITY For COACTIONS). $j$ is an isomorphism from $\left(A \# k[G]^{*}\right) * G$ to End $_{A}\left(A \# k G^{*}\right)$ which is isomorphic to $\operatorname{Mat}_{n}(A)$.

Remarks. If the standard basis of $A \# k G^{*},\left\{1 \# p_{g} \mid g\right.$ in $\left.G\right\}$, is chosen, one sees that for $a$ in $A, j(a \# 1)$ has matrix $\left(a_{i k^{-1}} \# 1\right)$, where $a_{j}$ is the $g_{j}$ homogeneous part of $a$ by using the formula [1, Proposition 1.4] $\sum_{i}\left(1 \# p_{i}\right)\left(a_{i k^{-1}} \# 1\right)=(a \# 1)\left(1 \# p_{k}\right)$. So $A_{1}$ (the component of 1 ) is embedded along the diagonal. Also $k G^{*}$ sits in $\operatorname{Mat}_{n}(A)$ as the idempotents $e_{i}$ which has a 1 in the $i-i$ position and is 0 elsewhere. Finally, $G$ is embedded via the regular representation. This was independently observed by D. Quinn [6, Theorem 1.3].

2. Inner and outer actions of $A \# k G^{*}$. An important object in the study of finite group actions of a semiprime ring $R$ is the algebra of the group $B(R, G)$. It is defined as follows: Let $g \in G, Q$ the maximal quotient ring of $R$, let $J_{g}=\{q \in$ $Q \mid x q=q x^{g}$ for all $\left.x \in R\right\}$; then $B=\sum J_{g}$.

Parts (1) and (2) of the next proposition are well known. 
TheOREM 3. Assume $R$ is $G$-Galois over $R^{G}$. Then

(1) If $L$ is a G-invariant left ideal of $R$ such that $\operatorname{tr}(L)=0$, then $L=0$.

(2) If $R^{G}$ is semiprime, then $R$ is semiprime.

(3) If $R^{G}$ is semiprime, then $B(R, G)$ is semiprime.

Proof. Let $a_{1}, \ldots, a_{t}, b_{1}, \ldots, b_{t}$ be a Galois basis. Now take $L$ as in (1). The Galois basis property implies for each $l$ in $L, l=\sum_{i} a_{i} \operatorname{tr}\left(b_{i} l\right)$, but this makes $l=0$.

(2) Let $D$ be a nilpotent left ideal of $R$, let $E=\Sigma_{G} D^{g}$. Then $\operatorname{tr}(E)$ is nilpotent; hence $D=0$.

To prove (3) we work in $Q$, the maximal quotient ring of $R$. By Kitamura's result [4] $Q$ is a $G$-Galois extension of $Q^{G}$ and $Q^{G}$ is the maximal quotient ring of $R^{G}$. Let $C$ be a $G$-invariant ideal of $B$ such that $C^{2}=0$. Let $D$ be the annihilator of $C$ in $Q^{G}$. Since $B$ commutes with $Q^{G}, D$ is the left and right annihilator of $C$. Also $\operatorname{tr}(Q C) \leqslant D$ and $(\operatorname{tr} Q C) D=0$ since $C$ is $G$-invariant and nilpotent. Thus $[\operatorname{tr} Q C]^{2}$ $=0$; since $Q^{G}$ is semiprime, (1) implies $C=0$. But if $E$ is any nilpotent ideal of $B$, then $C=\sum_{G} E^{g}$ is a $G$-invariant nilpotent ideal; hence $E=0$.

REMARKS. (1) We recall a result of Kharchenko: Let $G$ be a finite group. Then if $R$ and $B(R, G)$ are semiprime, then $R^{G}$ is semiprime [4, Theorem 6.1]. Thus Theorem 3 shows the converse holds if $R$ is $G$-Galois over $R^{G}$.

(2) Let $Q(R)=Q$ be the maximal quotient ring of $R$ or the Martindale quotient ring of $R$. Assume $R$ is $G$-Galois over $R^{G}$ and $R^{G}$ is semiprime. Theorem 3 says the standing hypotheses of [3] hold; namely $R$ and $B(R, G)$ are semiprime. Thus in the Martindale quotient ring case, $Q^{G}$ is the Martindale ring of quotients of $R^{G}[3$, Theorem 6.4]. Also $R^{G}$ is prime if and only if $B(R, G)$ is $G$-simple, by [3, Theorem 6.1 and Proposition 6.5]. In the case of the maximal ring of quotients, $Q^{G}$ is the maximal ring of quotients of $R^{G}$, as was pointed out in the proof of Theorem 3 . Finally, we note $B$ is the centralizer of $R^{G}$ in $Q$ [3, Theorem 6.2].

Thus, in our case, $A$ semiprime implies $B=B\left(A \# k G^{*}, G\right)$ is semiprime. We note that $A$ graded semiprime does not imply $B$ semiprime. For assume it did; then by [1, Theorem 2.9], $A \# k G^{*}$ is semiprime, but $B$ is semiprime, so by [4, Theorem 6.1] $A$ is semiprime. Thus, graded semiprime would imply semiprime, but this is not true.

I would like to thank the referee for improving the next result.

THEOREM 4. Assume that $G$ is finite and that $A$ is a semiprime G-graded ring. With $Q($ ) denoting either the left Martindale quotient ring or the maximal left quotient ring, then $Q(A)$ is a G-graded ring and $Q\left(A \# k G^{*}\right)$ is isomorphic, as a $k$-algebra, to $Q(A) \# k G^{*}$. Moreover, if $A$ is strongly $G$-graded, then $Q(A)$ is also.

Proof. Set $R=A \# k G^{*}$. We know $R$ is semiprime [1, Theorem 2.9] and has a $G$-action for which $R^{G}=A \# 1$. As is well known, this action extends to an action on each of the two quotient rings.

The second remark following Theorem 3 shows that $Q(R)^{G}$ is $Q(A)$. In either case, denote $Q(R)$ by $Q$ and $Q(R)^{G}$ by $S$. Then $Q$ is a ring with $G$-action, and $Q$ contains the idempotents $1 \# p_{g}$ for all $g$ in $G$. 
To see that $S$ is $G$-graded we use the criterion given in [1, p. 240], and define a function $\beta: G \rightarrow \operatorname{End}_{k}(S)$ where for $g$ in $G$ and $s \in S$,

$$
\beta(g)[s]=\operatorname{tr}\left[\left(1 \# p_{1}\right) s\left(1 \# p_{g^{-1}}\right)\right],
$$

where $\operatorname{tr} x=\Sigma_{G} x^{g}$ for $x \in Q$. Routine calculations verify the three properties on $\mathrm{p}$. 240 of [1]. So we have a grading on $S$ with $\beta(g)[s]$ as the $g$-component of $s$.

So we can form the smash product of $S$ and $k G^{*}$ and, to avoid confusion between operations in the smash product of $S$ and $k G^{*}$ and in the ring $Q=Q\left(A \# k G^{*}\right)$, we temporarily use $\$$ to denote the former. In other words, elements of $S \$ k G^{*}$ are sums of terms of the form $s \$ p_{g}$ with $s \in S, g \in G$. Define a homomorphism $\phi$ : $S \$ k G^{*}$ into $Q$ where $\phi\left(s \$ p_{g}\right)=s\left(1 \# p_{g}\right)$ where this latter product makes sense in $Q$. That $\phi$ is well defined follows since $S \$ k G^{*}$ is a free $S \$ 1$ module. To see that $\phi$ is one-to-one, suppose that $\sum_{G} s_{g}\left(1 \# p_{g}\right)=0$.

There is a member $D$ of the appropriate filter of ideals or left ideals of $A$ with each ( $D \# 1) s_{g}$ contained in $A \# 1$. So $\sum_{G}(D \# 1) s_{g}\left(1 \# p_{g}\right)=0$; thus $(D \# 1) s_{g}=0$, in $A \# 1$ and $s_{g}=0$ in $S$. To show $\phi$ is a ring homomorphism one must recall how $S$ is graded. So let $s, t \in S$ and $g, h \in G$; then

$$
\begin{aligned}
\phi[(s & \left.\left.\$ p_{g}\right)\left(t \$ p_{h}\right)\right]=s t\left(g h^{-1}\right)\left(1 \# p_{h}\right) \\
& =s \operatorname{tr}\left[\left(1 \# p_{1}\right) t\left(1 \# p_{h g^{-1}}\right)\right]\left(1 \# p_{h}\right) \\
& =s\left(1 \# p_{g}\right) t\left(1 \# p_{h}\right)=\phi\left(s \$ p_{g}\right) \phi\left(t \$ p_{h}\right) .
\end{aligned}
$$

Finally to see $\phi$ is onto, let $q \in Q$. For each $x \in G$, set $q_{x}=\operatorname{tr}\left(q\left(1 \# p_{x}\right)\right)$, this being fixed, so is in $S$. Now $q\left(1 \# p_{x}\right)=q_{x}\left(1 \# p_{x}\right)$ so $q=\Sigma_{x} q\left(1 \# p_{x}\right)$ is in $\Sigma_{x} S\left(1 \# p_{x}\right)$. Thus $\phi$ is a $k$-algebra isomorphism. To show $S$ is strongly graded, if $A$ is also, we need to show the ideal $S\left(1 \# p_{1}\right) S$ equals $S \# k G^{*}$, but since $A$ is strongly graded, $A\left(1 \# p_{1}\right) A=A \# k G^{*}$. Here we are using [1, Theorem 2.12].

REMARK. See [5, Theorem 5.1]. The definitions of $X$-inner and $X$-outer are in [3]. Let $G_{\text {inn }}=\left\{g \in G \mid g\right.$ is $X$-inner on $\left.A \# k G^{*}\right\}$. Now if $A \# k G^{*}$ is prime, then $G_{\text {inn }}$ is a normal subgroup of $G$.

TheOREM 5. Let $A$ be semiprime and G-graded. Assume $G_{\text {inn }}$ is a subgroup of $G$. With $Z(\quad)$ denoting the center of a ring, then $Z(A)$ is contained in $\sum_{h} A_{h}$, with $h$ in $G_{\text {inn }}$. In particular, if $G$ is $X$-outer, then $Z(A)$ is contained in $A_{1}$.

Proof. We denote $\mathrm{G}_{\text {inn }}$ by $H$ and $A \# k G^{*}$ by $R$. Since $R^{G}=A$ is semiprime, $B(R, G)$ is too by Theorem 3. By the definition of the algebra of the group, it follows that $B(R, G)=B(R, H)$ : call it $B$. We let $Q$ be the Martindale quotient ring of $R$, so [3, Theorem 6.2] gives that $B$ is the centralizer in $Q$ of both $R^{H}$ and $R^{G}$. Now it follows easily that $Z(A) \leqslant Z\left(R^{H}\right)$. Let $f_{1}=\Sigma_{H} 1 \# p_{h}$, which is an element of $R^{H}$. So if $a \# 1$ is in $Z(A)$, then $(a \# 1) f_{1}=f_{1}(a \# 1)$. Upon equating the coefficients of $1 \# p_{1}$, we see $Z(A) \leqslant \sum_{H} A_{h}$.

S. Montgomery has an unpublished example that show the converse is false.

\section{REFERENCES}

1. M. Cohen and S. Montgomery, Group-graded rings, smash products, and group actions, Trans. Amer. Math. Soc. 282 (1984), 237-258.

2. F. R. DeMeyer, Some notes on the general Galois theory of rings, Osaka J. Math. 2 (1965), 117-127. 
3. J. M. Goursaud, J. L. Pascaud and J. Valette, Sur les travaux de V. K. Kharchenko, Lecture Notes in Math., vol. 924, Springer-Verlag, Berlin and New York, pp. 322-354.

4. Y. Kitamura, Note on the maximal quotient ring of a Galois subring, Math. J. Okayama Univ. 19 (1976-1977), 55-60.

5. C. Nastasecu, Strongly graded rings of finite groups, Comm. Algebra 11 (10), (1983), 1033-1071.

6. D. Quinn, Group-graded rings and duality, Trans. Amer. Math. Soc. 292 (1985), 155-167.

7. M. van den Bergh, "A duality theorem for Hopf algebras", Methods in ring theory, Reidel, 1984.

Department of Mathematical Sciences, University of Cincinnati, Cincinnati, Ohio 45221 0025 